\title{
Pseudogap and Kinetic Pairing Under Critical Differentiation of Electrons in Cuprate Superconductors
}

\author{
Masatoshi Imada ${ }^{1)}$ and Shigeki Onoda \\ Institute for Solid State Physics, University of Tokyo, 5-1-5, Kashiwanoha, Kashiwa, \\ Chiba, 277-8581, Japan \\ 1) e-mail address, imada@issp.u-tokyo.ac.jp
}

\begin{abstract}
Superconducting mechanism of cuprates is discussed in the light of the proximity of the Mott insulator. The proximity accompanied by suppression of coherence takes place in an inhomogeneous way in the momentum space in finite-dimensional systems. Studies on instabilities of metals consisted of such differentiated electrons in the momentum space are reviewed from a general point of view. A typical example of the differentiation is found in the flattening of the quasiparticle dispersion discovered around momenta $(\pi, 0)$ and $(0, \pi)$ on $2 \mathrm{D}$ square lattices. This flattening even controls the criticality of the metal-insulator transition. Such differentiation and suppressed coherence subsequently cause an instability to the superconducting state in the second order of the strong coupling expansion. The d-wave pairing interaction is generated from such local but kinetic processes in the absence of disturbance from the coherent single-particle excitations. The superconducting mechanism emerges from a direct kinetic origin which is conceptually different from the pairing mechanism mediated by bosonic excitations as in magnetic, excitonic, and BCS mechanisms. Pseudogap phenomena widely observed in the underdoped cuprates are then naturally understood from the mode-mode coupling of d-wave superconducting (dSC) fluctuations repulsively coupled with antiferromagnetic (AFM) ones. When we assume the existence of a strong d-wave channel repulsively competing with AFM fluctuations under the formation of flat and damped single-particle dispersion, we reproduce basic properties of the pseudogap seen in the magnetic resonance, neutron scattering, angle resolved photoemission and tunneling measurements in the cuprates.
\end{abstract}

\section{Introduction}

Magnetism in strongly correlated electron systems has been a subject of extensive studies for a long time. In many examples, the energy gain from electron kinetic energy term is a crucial driving force for stabilizing magnetically symmetry broken states. Even in the Mott insulating state, this is equally true. Although the itinerancy of electrons is lost by the Coulomb interaction in the Mott insulator, electrons still find an optimized way to gain the energy from the kinetic energy term through a virtual process, where the strong coupling expansion becomes an adequate description under totally suppressed coherent 
motion of electrons. Anderson's mechanism of the superexchange interaction thus proposed [1 has long been one of the most fruitful concepts in the condensed matter physics. The double exchange mechanism proposed by de Gennes [2], Zenner [3] and Anderson and Hasegawa [4] is another example, where a ferromagnetic (FM) metal is stabilized by the kinetic energy gain under a strong Hund's rule coupling between degenerate orbitals.

Superconductivity, on the other hand, has been analyzed mainly from a different view since the dramatic success of BCS theory [5]. Various types of pairing interactions mediated by bosonic excitations have been considered to be responsible for the mechanisms. To say nothing of the electron-phonon interaction, roles of AFM and FM paramagnons and excitons have been studied in various contexts. In these proposals, the superconducting ground state is the consequence of pair formation by the mediated attraction in the presence of coherent and metallic quasiparticles.

In this report, we discuss in more general perspective the importance of the kinetic origin for various symmetry breakings. We particularly discuss the kinetic mechanism of the superconductivity under the suppressed coherence of quasiparticles near the Mott insulator. The mechanism of superconductivity we discuss in this paper is conceptually different from the conventional magnetic mechanism discussed in the spin fluctuation theories and in the $t-J$ model, because the origin is not in the mediated bosons but in the direct kinetic process as in the superexchange for the mechanism of antiferromagnetism. Although the superexchange interaction is the only process for the energy lowering by the kinetic energy term in the Mott insulating phase, this constraint is lost in the presence of doped carriers and various other processes may equally or even more importantly contribute to the kinetic energy gain. The existence of various other processes is in fact easily confirmed by the strong coupling expansion of the Hubbard-type models, where in the second order, namely in the order of $t^{2} / U$, it not only generates the superexchange interaction but also other two-particle terms including the pair hopping processes. Here in the strong coupling expansion, the onsite Coulomb repulsion $U$ is assumed to be larger than the kinetic transfer term $t$. These second-order terms usually have secondary importance in the presence of the kinetic energy term in the first order of $t$ in the normal metal. However, as similarly to the superexchange interaction in the Mott insulating phase, they become the primary origin of the kinetic energy gain even in metals when the singleparticle processes are suppressed by some reason but the amplitude of the second-order terms are retained. In the study on the strong-coupling expansion of the d-p model, it was shown that the d-wave pair-hopping process appears in the third order, although the superexchange interaction appears only in the fourth order [6]. In the next section we first discuss the origin of the unusual suppression of coherent single-particle motion in the proximity of the Mott insulator before considering the mechanism of superconductivity.

High-temperature cuprate superconductors show a variety of unusual properties in the 
normal state [7]. Among others, we concentrate on two remarkable properties widely observed in the cuprates for the purpose of examining the relevance of the kinetic mechanism as the driving mechanism of the high-Tc superconductivity. One of the remarkable properties in the cuprates is the flat dispersion in single-particle excitations. Angle resolved photoemission spectra (ARPES) in $\mathrm{Y}$ and Bi based high-Tc cuprates show an unusual dispersion which is far from weak correlation picture [8]. The dispersion around $(\pi, 0)$ and $(0, \pi)$ is extremely flat beyond the expectations from usual van Hove singularities. The flat dispersion also shows rather strong damping.

The other remarkable property we discuss is the pseudogap phenomenon observed in the underdoped region [7, 9]. It is observed both in spin and charge excitations in which gap structure emerges from a temperature $T_{\mathrm{PG}}$ well above the superconducting transition point $T_{\mathrm{c}}$. The gap structure is observed in various different probes such as NMR relaxation time, the Knight shift, neutron scattering, tunneling, ARPES, specific heat, optical conductivity, and DC resistivity. The ARPES [10, 11] data have revealed that the pseudogap starts growing first in the region around $(\pi, 0)$ and $(0, \pi)$ from $T=T_{\mathrm{PG}}$ much higher than $T_{c}$. Therefore, the pseudogap appears from the momentum region of the flattened dispersion and it is likely that the mechanism of the pseudogap formation is deeply influenced from the underlying flatness. The superconducting state itself also shows a dominant gap structure in this flat spots, $(\pi, 0)$ and $(0, \pi)$, due to the $d_{x^{2}-y^{2}}$ symmetry. In fact, the pseudogap structure above $T_{c}$ appears continuously to merge into the $d_{x^{2}-y^{2}}$ gap below $T_{c}$. To understand the superconducting mechanism and the origin of the high transition temperatures, a detailed understanding of the physics taking place in the flat dispersion region is required.

The emergence of the flat dispersion around $(\pi, 0)$ and $(0, \pi)$ has also been reported in numerical simulation results rather universally in models for strongly correlated electrons such as the Hubbard and $t-J$ models on square lattices [12, 13, 14. As we see in the next section, this criticality is interpreted from a strong proximity of the Mott insulator where strong electron correlation generates suppressed dynamics and coherence.

\section{Kinetic Pairing Derived from Electron Differenti- ation}

In a simple picture, the correlation effects emerge as the isotropic mass renormalization, where the Coulomb repulsion from other electrons makes the effective mass heavier. This effect was first demonstrated by Brinkman and Rice [19] in the Gutzwiller approximation and refined in the dynamical mean field theory [20].

In the numerical results on a square lattice, as discussed above, the correlation effects appear in more subtle way where the electrons at different momenta show different 
renormalizations. When the Mott insulator is approached and the doping concentration becomes small, the mass renormalization generally becomes stronger. However, once the renormalization effect gets relatively stronger in a part of the Fermi surface, it is further enhanced at that part in a selfconsistent fashion because the slower electrons become more and more sensitive to the correlation effect. This generates critical differentiation of the carriers depending on the portion of the Fermi surface.

On square lattices, the stronger renormalization happens around $(\pi, 0)$ and $(0, \pi)$. A part of this anisotropic correlation effect concentrating near $(\pi, 0)$ and $(0, \pi)$ is intuitively understood from the carrier motion under the background of AFM correlations. As we see a real space picture in Fig.1a, the carrier motion in the diagonal directions does not disturb the correlations due to the parallel spin alignment, while the motion in horizontal and vertical directions strongly disturbs the AFM backgrounds as expressed as the wavy bonds and the motion itself is also disturbed as a feedback. Such strong coupling of charge dynamics to spin correlations causes flattening and damping of electrons around $(\pi, 0)$ and $(0, \pi)$, but not around the diagonal direction $( \pm \pi / 2, \pm \pi / 2)$. The anisotropic renormalization effect eventually may generate a singularly flat dispersion on particular region of the Fermi surface, which accepts more and more doped holes in that region due to the enhanced density of states.

The transition to the Mott insulator is then governed by that flattened part, since the carriers reside predominantly in the flat region. The criticality of the metal-insulator transition on the square lattice is thus determined from the doped carriers around the flat spots, $(\pi, 0)$ and $(0, \pi)$. The hyperscaling relation becomes naturally satisfied because singular points on the momentum space govern the transition. In fact, the hyperscaling relations are numerically supported in various quantities and shows agreements with experimental indications. For example, the electronic compressibility critically diverges as $\kappa \propto 1 / \delta$ with decreasing doping concentration $\delta$ while the Drude weight is unusually suppressed as $D \propto \delta^{2}$ [15, 16]. The coherence temperature (the effective Fermi temperature) is also scaled as $T_{F} \propto \delta^{2}$ and indicates unusual suppression. In more comprehensive understanding, all the numerical data are consistent with the hyperscaling relations with a large dynamical exponent $z=4$ for the metal-insulator transition [7, 18]. Such large exponent opposed to the usual value $z=2$ for the transition to the band insulator is derived from the slower electron dynamics even at $T=0$ generated by the flat dispersion.

We, however, should keep in mind that the relaxation time of quasiparticles and the damping constant of magnetic excitations do not have criticality at the transition point to the Mott insulator. A general remark is that the relaxation time is critical only in the case of the Anderson localization transition and not in the case of the transition to the Mott insulator. The DC transport properties and magnetic relaxation phenomena are contaminated by such noncritical relaxation times $\tau$ and are influenced by the carriers 
in the other portion than the flat part because the flat part has stronger damping and less contributes to the DC properties. Large anisotropy of $\tau$ masks the real criticality and makes it difficult to see the real critical exponents in the $\tau$-dependent properties. Relevant quantities to easily estimate the criticality is the $\tau$ independent quantities such as the Drude weight and the compressibility.

Near the metal-insulator transition, the critical electron differentiation and selective renormalization may lead to experimental observations as if internal degrees of freedom of the carriers such as spin and charge were separated because each degrees of freedom can predominantly be conveyed by carriers in different part of the Fermi surface. Another possible effect of the electron differentiation is the appearance of several different relaxation times which are all originally given by a single quasiparticle relaxation in the isotropic Fermi liquids, but now depend on momenta of the quasiparticles.

Another aspect one might ask in connection to the relevance of the flat part to the metal-insulator transition is the observed level difference between $(\pi, 0)$ and $( \pm \pi / 2, \pm \pi / 2)$ in the undoped and underdoped cuprates [17]. The level at $(\pi, 0)$ is substantially far from the Fermi level, and at a first glance, it does not have a chance to contribute to the metalinsulator transition. However, it has been clarified [6] that this level difference is likely to be absent before the d-wave interaction channel starts growing where the flat part indeed governs the criticality, while it is developed by renormalization of the single-particle level accompanied by the d-wave interaction in the lower energy scale. It is remarkable that this renormalization exists even in the insulator.

If the mass renormalization would happen in an isotrpic way as in the picture of Brinkman and Rice, the renormalization can become stronger without disturbance when the insulator is approached. However, if the singularly renormalized flat dispersion emerges critically only in a part near the Fermi surface but the whole band width is ratained, that flattened part has stronger instability due to the coupling to larger energy scale retained in other part of the momentum space. The instability can be mediated by local and incoherent carrier motion generated from two-particle processes derived in the strong coupling expansion [6]. The local two-particle motion is given in the order of $t^{2} / U$ with the bare $t$ while the single-particle term is renormalized to $t^{*}$ which can be smaller than $t^{2} / U$ at the flattened portion. The instability of the flat dispersion was studied by taking account such local and incoherent terms in the Hubbard and $t-J$ models [21, 22, [16]. The inclusion of the two-particle terms drives the instability of the flat part to the superconducting pairing and the formation of the d-wave gap structure. In fact, even at half filling, the two-particle process stabilizes the d-wave superconducting state and reproduces the basic feature of the pseudogap formation 21, 22 observed in the BEDT-TTF compounds 23].

The paired bound particles formed from two quasiparticles at the flat spots have dif- 
ferent dynamics from the original quasiparticle. In fact, when the paired singlet becomes the dominant carrier, the criticality changes from $z=4$ to $z=2$, resulting in the recovery of coherence and kinetic energy gain [16]. It generates a strong pairing interaction from the kinetic origin. This pairing mechanism is a consequence of suppressed single-particle coherence and electron differentiation due to strong correlations.

The instability of the flat dispersion coexisting with relatively large incoherent process was further studied [6, 24, 25, 26]. It has turned out that promotion of the above scaling behavior and the flat dispersion offers a way to control potential instabilities. Even when a flat band dispersion is designed near the Fermi level by controlling lattice geometry and parameters, it enlarges the critical region under the suppression of single-particle coherence in the proximity of the Mott insulator mentioned above. In designed lattices and lattices with tuned lattice parameters, it was reported that the superconducting instability and the formation of the spin gap have been dramatically enhanced [24].

\section{Pseudogap Phenomena in Cuprates as Supercon- ducting Fluctuations}

As is mentioned in $\S 1$, the pseudogap in the high-Tc cuprates starts growing from the region of the flat dispersion. When the single-particle coherence is suppressed, the system is subject to two particle instabilities. As clarified in $\S 2$, the superconducting instability in fact grows. However, the AFM and charge order correlations are in principle also expected to grow from other two-particle (particle-hole) processes and may compete each other. In particular, the AFM long-range order is realized in the Mott insulator and its short-range correlation is well retained in the underdoped region. Therefore, to understand how the superconducting phase appears in the underdoped region, at least competition of dSC and AFM correlations has to be treated with underlying suppressed coherence in the region of $(\pi, 0)$ and $(0, \pi)$. The authors have developed a framework to treat the competition by employing the mode-mode coupling theory of dSC and AFM fluctuations where these two fluctuations are treated on an equal footing [27, 28].

It should be noted that the strong dSC pairing interaction is resulted from a highly correlated effect with electron differentiation while the critical differentiation has not been successfully reproduced from the diagrammatic approach so far. Then, within the framework of the mode-mode coupling theory, at the starting point, we have assumed the existence of correlation effects leading to the flattened dispersion and the $d$-wave pair hopping process. The AFM and dSC fluctuations are predominantly generated by the contributions from the quasiparticle excitations in the flattened regions $(\pi, 0)$ and $(0, \pi)$. These fluctuations are treated in a set of selfconsistent equations with mode couplings of dSC and AFM. From the selfconsistent solution, the pseudogap formation is well repro- 
duced in a region of the parameter space. The pseudogap emerges when the mode coupling between dSC and AFM is repulsive with a severe competition and dSC eventually dominates at low temperatures. Such competition suppresses $T_{c}$, while above $T_{c}$ it produces a region where pairing fluctuations are large. This region at $T_{P G}>T>T_{c}$ shows suppression of $1 / T_{1} T$ and the pseudogap formation around $(\pi, 0)$ and $(0, \pi)$ in $A(k, \omega)$. These reproduce the basic feature of the pseudogap phenomena experimentally observed in the underdoped cuprates. The pseudogap formation is identified as coming from the superconducting fluctuations. The momentum dependence shows that the pseudogap formation starts around $(\pi, 0)$ from higher temperatures and the formation temperature becomes lower with increasing distance from $(\pi, 0)$. All of the above reproduce the experimental observations.

We, however, note a richer structure of the gap formation observed in the transversal NMR relaxation time $T_{2 G}$ and the neutron resonance peak. One puzzling experimental observation is that the pseudogap structure appears in $1 / T_{1} T$ [9, 29, 30, 31, 32], while in many cases $1 / T_{2 \mathrm{G}}$, which measures $\operatorname{Re} \chi(Q, \omega=0)$ at $Q=(\pi, \pi)$, continuously increases with the decrease in temperature with no indication of the pseudogap. In addition, the so called resonance peak appears in the neutron scattering experiments [33]. A resonance peak sharply grows at a finite frequency below $T_{\mathrm{c}}$ with some indications even at $T_{c}<$ $T<T_{\mathrm{PG}}$. This peak frequency $\omega^{*}$ decreases with lowering doping concentration implying a direct and continuous evolution into the AFM Bragg peak in the undoped compounds. The neutron and $T_{2 \mathrm{G}}$ data support the idea that the AFM fluctuations are suppressed around $\omega=0$ but transferred to a nonzero frequency below $T_{\mathrm{PG}}$.

To understand these features, a detailed consideration on damping of the magnetic excitations is required. With the increase in the pairing correlation length $\xi_{d}$, the pseudogap in $A(k, \omega)$ is developed. Since the damping is mainly from the overdamped Stoner excitations, the gap formation in $A(k, \omega)$ contributes not only to suppress growth of AFM correlation length $\xi_{\sigma}$ but also to reduce the magnetic damping because, inside the domain of the $d$-wave order, the AFM excitations are less scattered due to the absence of lowenergy quasiparticle around $(\pi, 0)$. If the quasiparticle damping is originally large around $(\pi, 0)$, the damping $\gamma$ can be reduced dramatically upon the pseudogap formation. Under this circumstance, our calculated result reproduces the resonance peak and the increase in $1 / T_{2 G}$ with lowering temperature at $T>T_{c}$ in agreement with the experimental observations in $\mathrm{YBa}_{2} \mathrm{Cu}_{3} \mathrm{O}_{6.63}, \mathrm{YBa}_{2} \mathrm{Cu}_{4} \mathrm{O}_{8}$ and some other underdoped compounds [27, 28].

A subtlety arises when the damping around $(\pi / 2, \pi / 2)$ starts contributing. This is particularly true under the pseudogap formation. If contributions from the $(\pi / 2, \pi / 2)$ region would be absent, the damping of the magnetic excitation would be strongly reduced when the pseudogap is formed around $(\pi, 0)$ as we mentioned above. However, under the pseudogap formation, the damping can be determined by the Stoner continuum generated 
from the $(\pi / 2, \pi / 2)$ region and can remain overdamped. This process is in fact important if the quasiparticle damping around the $(\pi / 2, \pi / 2)$ region is large as in the case of La 214 compounds [34. The formation of the pseudogap itself is a rather universal consequence of the strong coupling superconductors. However, the actual behavior may depend on this damping. If the damping generated by the $(\pi / 2, \pi / 2)$ region is large, it sensitively destroy the resonance peak structure observed in the neutron scattering experimental results.

\section{Conclusion and Discussion}

Electron critical differentiation is a typical property of the proximity of the Mott insulator. The flattening of the quasiparticle dispersion appears around momenta $(\pi, 0)$ and $(0, \pi)$ on square lattices and determines the criticality of the metal-insulator transition with the suppressed coherence in that momentum region of quasiparticles. Such coherence suppression subsequently causes an instability to the superconducting state when a proper incoherent kinetic process is retained. The d-wave superconducting state is stabilized from such retained microscopic process derived from the strong correlation expansion. The origin of the superconductivity is ascribed to the kinetic energy gain.

By assuming the d-wave channel and the presence of strongly renormalized flat quasiparticle dispersion around the $(\pi, 0)$ region, we have constructed the mode-mode coupling theory for the AFM and $d \mathrm{SC}$ fluctuations. The pseudogap in the high- $T_{\mathrm{c}}$ cuprates is reproduced as the region with enhanced $d \mathrm{SC}$ correlations and is consistently explained from precursor effects for the superconductivity. The existence of the flat region plays a role to suppress the effective Fermi temperature $E_{F}$. This suppressed $E_{F}$ and relatively large local pair hopping process both drive the system to the strong coupling superconductor thereby leading to the pseudogap formation. The pseudogap formation is also enhanced by the AFM fluctuations repulsively coupled with dSC fluctuations.

Several similar attempts have also been made to reproduce the pseudogap phenomena observed in the cuprates. A common conclusion inferred from these calculations including those by the present authors is that the pseudogap is reproduced when the d-wave channel is explicitly assumed [27, 28, 35] while crucial features such as the pseudogap formation around $(\pi, 0)$ cannot be well reproduced if one tries to derive the superconducting channel itself from the AFM spin fluctuations [36]. This difficulty is summarized: if one desires to stabilize a strongly fluctuating pseudogap region well above $T_{c}$ generated originally by the magnetic interaction, one has to treat the strong-coupling superconductivity and hence even more strong-coupling magnetic interaction. In such a circumstance, it is dificult to escape from the magnetic instability before the pseudogap formation. The difficulty is naturally interpreted from our analysis: The kinetic d-wave channel we derived in the strong coupling expansion is not ascribed to the magnetic origin and is not contained 
neither in the spin-fluctuation mechanism nor in the $t$ - $J$ model. This superconducting channel has a comparable amplitude to the magnetic one, namely $t^{2} / U$. Furthermore the channel is enhanced by the flattened dispersion and the electron critical differentiation. Such effects are far beyond the one-loop level of the weak coupling approach. The change in the quasiparticle dispersion in the portion of the Fermi surface is not well reproduced for the moment and the momentum dependent selfenergy appears to be significantly underestimated in the existing diagrammatic evaluations.

The strong coupling Hamiltonian was considered before [37 and also in terms of the pairing arising from the attractive superexchange interaction 38. The superconductivity from direct kinetic origin was also discussed before in a specific model [39 as well as in the interlayer tunneling mechanism [40], where interlayer kinetic energy gain is required to stabilize the superconductivity. If we identify the intralayer charge incoherence observed in the normal state as the crossover phenomena above the unusually suppressed Fermi temperature in the proximity of the Mott insulator, and notice that no quantum coherence is reached even within the layer, the kinetic origin of the superconductivity is found solely in a two-dimensional plane.

Under the suppression of coherence in the proximity of the Mott insulator, the secondorder process in the strong-coupling expansion may also drive other type of ordered state in addition to the superconductivity. In particular, because the charge compressibility is enhanced due to the flattened dispersion, the charge fluctuation may be strongly en-

hancced and the charge ordering and stripes can be triggered both by the kinetic energy gain and the intersite Coulomb interaction. When the competition between the superconductivity and the charge ordering becomes serious, presumable repulsive mode coupling of them may become another origin of the pseudogap formation, although such evidence is not clear in the present experimental results of the cuprates.

The presend theory of the kinetic superconductor predicts a specific form of the kinetic energy gain to be seen in the optical conductivity [41] and the single particle spectra 442. Qualitatively, it is expected that the in-plane kinetic energy starts gained even in the pseudogap region. The energy gain dominantly coming from the $(\pi, 0)$ and $(0, \pi)$ region of the single particles must have a significant doping dependence associated to the change in the dynamical exponent $z$ from 4 to 2 .

\section{References}

[1] P.W. Anderson: Phys. Rev. 115 (1959) 2.

[2] P.G. deGennes: Phys. Rev. 118 (1960) 141.

[3] C. Zener: Phys. Rev. 82 (1951) 403.

[4] P.W. Anderson and H. Hasegawa: Phys. Rev. 100 (1955) 675. 
[5] J. Bardeen, L. N. Cooper and J.R. Schrieffer: Phys. Rev. 108 (1957) 1175

[6] H. Tsunetsugu and M. Imada: J. Phys. Soc. Jpn. 68 (1999) 3162.

[7] For a recent review see M. Imada, A. Fujimori and Y. Tokura: Rev. Mod. Phys. 70 (1998) 1039, Sec. IV.C.

[8] K. Gofron, J. C. Campuzano, A. A. Abrikosov, M. Lindroos, A. Bansil, H. Ding, D. Koelling and B. Dabrowski: Phys. Rev. Lett. 73 (1994) 3302. D. S. Marshall, D. S. Dessau, A. G. Loeser, C-H. Park, A. Y. Matsuura, J. N. Eckstein, I. Bozovic, P. Fournier, A. Kapitulnik, W. E. Spicer and Z.-X. Shen: Phys. Rev. Lett. 76 (1996) 4841.

[9] H. Yasuoka, T. Imai and T. Shimizu: "Strong Correlation and Superconductivity" ed. by H. Fukuyama, S. Maekawa and A. P. Malozemoff (Springer Verlag, Berlin, 1989), p.254.

[10] Z.-X. Shen and D. S. Dessau: Physics Reports 253 (1995) 1; A. G. Loeser, Z.-X. Shen, D. S. Dessau, D. S. Marshall, C. H. Park, P. Fournier and A. Kapitulnik: Science 273 (1996) 325.

[11] H. Ding, T. Yokoya, J. C. Campuzano, T. Takahashi, M. Randeria, M. R. Norman, T. Mochiku, K. Kadowaki and J. Giapintzakis: Nature 382 (1996) 51.

[12] E. Dagotto, A. Nazarenko and M. Boninsegni: Phys. Rev. Lett. 73 (1994) 728.

[13] N. Bulut, D.J. Scalapino and S.W. White: Phys. Rev. B 50 (1994) 7215.

[14] F. F. Assaad and M. Imada: Eur. Phys. J. B 10 (1999) 595.

[15] N. Furukawa and M. Imada: J. Phys. Soc. Jpn. 61 (1992) 3331; ibid. 62 (1993) 2557.

[16] H. Tsunetsugu and M. Imada: J. Phys. Soc. Jpn. 67, (1998)1864.

[17] C. Kim et al.:Phys. Rev. B 56 (1998) 15589.

[18] M. Imada, J. Phys. Soc. Jpn.64(1995)2954.

[19] W.F.Brinkman, and T.M. Rice, Phys. Rev. B2,(1970) 4302.

[20] A.Georges, G. Kotliar, W. Krauth, and M. J. Rozenberg, Rev. Mod. Phys. 68(1996) 13.

[21] F.F. Assaad, M. Imada and D.J. Scalapino:Phys. Rev. B 56 (1998) 15001.

[22] F.F. Assaad and M, Imada: Phys. Rev. B. 58, (1998) 1845.

[23] K. Kanoda: Hyperfine Interact. 104 (1997) 235.

[24] M. Imada and M. Kohno:Phys. Rev. Lett. 84 (2000) 143.

[25] M. Imada, M. Kohno and H. Tsunetsugu: Physica B 280(2000) 303.

[26] M. Kohno and M. Imada: J. Phys. Soc. Jpn. 69(2000) 25.

[27] S. Onoda and M. Imada: J. Phys. Soc. Jpn. 68 (1999) 2762.

[28] S. Onoda and M. Imada: J. Phys. Soc. Jpn. 69 (2000) 312.

[29] H. Zimmermann, M. Mali, D.Brinkmann, J. Karpinski, E. Kaldis and S. Rusiecki: Physica C 159 (1989) 681; T. Machi, I. Tomeno, T. Miyataka, N. Koshizuka, S. Tanaka, T. Imai and H. Yasuoka: Physica C 173 (1991) 32. 
[30] K. Ishida, Y. Kitaoka, K. Asayama, K. Kadowaki and T. Mochiku: Physica C 263 (1996) 371.

[31] Y. Itoh, T. Machi, A. Fukuoka, K. Tanabe, and H. Yasuoka: J. Phys. Soc. Jpn. 65 (1996) 3751.

[32] M.-H. Julien, P. Carretta, M. Horvatić, C. Berthier, Y. Berthier, P. Ségransan, A. Carrington and D. Colson: Phys. Rev. Lett. 76 (1996) 4238.

[33] H. F. Fong, B. Keimer, D. L. Milius and I. A. Aksay: Phys. Rev. Lett. 78 (1997) 713.

[34] A. Ino, C. Kim, T. Mizokawa, Z.-X. Shen, A. Fujimori, M. Takabe, K. Tamasaku, H. Eisaki and S. Uchida: J. Phys. Soc. Jpn. 68 (1999) 1496; A. Ino, T. Mizokawa, K. Kobayashi, A. Fujimori, T. Sasagawa, T. Kimura, K. Kishio, K. Tamasaku, H. Eisaki, and S. Uchida: Phys. Rev. Lett. 78 (1998) 2124.

[35] Y. Yanase and K. Yamada: J. Phys. Soc. Jpn. 68 (1999) 2999.

[36] A. Kobayashi, A. Tsuruta, T. Matsuura and Y. Kuroda: J. Phys. Soc. Jpn. 68 (1999) 2506.

[37] K.A.Chao, J. Spalek and A.M. Oles: J. Phys. C 10 (1977) L271.

[38] J. E. Hirsch: Phys. Rev. Lett. 54 (1985) 1317.

[39] J. E. Hirsch: Physica C 201 (1992) 347.

[40] J.M. Wheatley, T.C. Hsu, and P.W. Anderson: Phys. Rev. Lett. 37 (1988) 5897.

[41] D.N. Basov et al.: Science 283 (1999) 49.

[42] N.R. Norman, M. Randeria, B. Janko and J. C. Campuzano: Phys. Rev. B 61 (2000) 14742. 


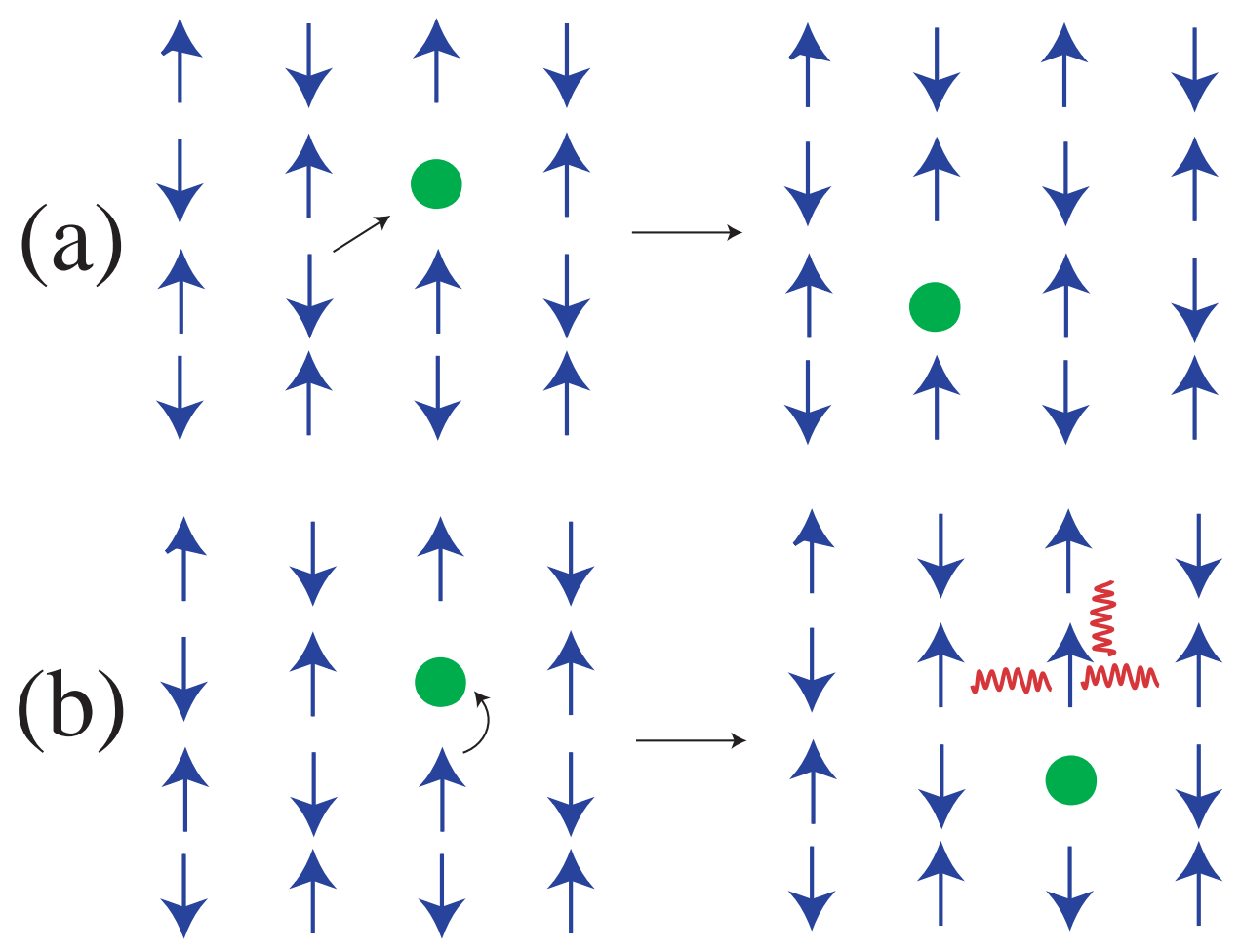

Figure 1: Intuitive picture to understand anisotropic renormalization effects. An electron moving in diagonal directions under the AFM correlations are not severely renormalized as in (a) while they are for horizontal or vertical directions as in (b). In (b), frustrations are generated after the hole motion (denoted by circles) as shown by wavy bonds. These differences induce the differentiation between electrons around $(\pi, 0)$ and $(\pi / 2, \pi / 2)$. 